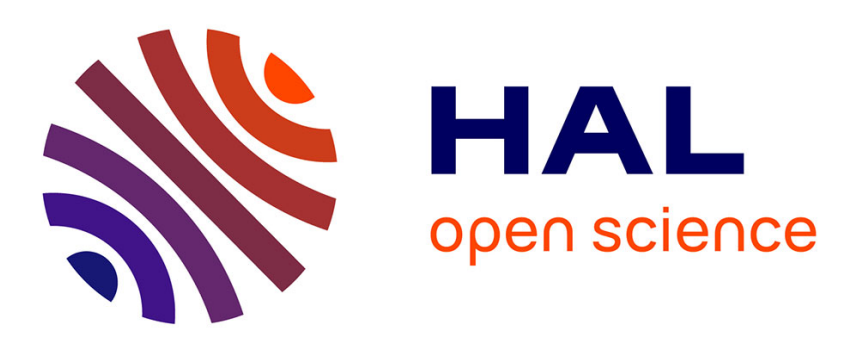

\title{
Méthode de consolidation des courbes de tarage pour les crues d'occurrence rare sur le bassin versant expérimental du Real Collobrier
}

M. Tolsa, Y. Aubert, J. Le Coz, Benjamin Renard, P. Arnaud, J.A. Fine, D. Organde

\section{To cite this version:}

M. Tolsa, Y. Aubert, J. Le Coz, Benjamin Renard, P. Arnaud, et al.. Méthode de consolidation des courbes de tarage pour les crues d'occurrence rare sur le bassin versant expérimental du Real Collobrier. La Houille Blanche - Revue internationale de l'eau, 2013, 6, pp.16-23. 10.1051/lhb/2013045 . hal01123868

\section{HAL Id: hal-01123868 \\ https://hal.science/hal-01123868}

Submitted on 6 Mar 2015

HAL is a multi-disciplinary open access archive for the deposit and dissemination of scientific research documents, whether they are published or not. The documents may come from teaching and research institutions in France or abroad, or from public or private research centers.
L'archive ouverte pluridisciplinaire HAL, est destinée au dépôt et à la diffusion de documents scientifiques de niveau recherche, publiés ou non, émanant des établissements d'enseignement et de recherche français ou étrangers, des laboratoires publics ou privés. 


\title{
Méthode de consolidation des courbes de tarage pour les crues d'occurrence rare sur le bassin versant expérimental du Real Collobrier
}

\author{
Mathieu TOLSA ${ }^{1}$, Yoann AUBERT², Jérôme LE COZ³ , Benjamin RENARD ${ }^{4}$, \\ Patrick ARNAUD ${ }^{1}$, Jean-Alain FINE ${ }^{2}$ et Didier ORGANDE ${ }^{2}$
}

\author{
Irstea, UR OHAX, Hydrologie - 3275 route Cézanne CS 40061, 13182 Aix-en-Provence - Cedex 5 - prenom.nom@irstea.fr \\ 2. HYDRIS-Hydrologie - 5 Avenue du grand chêne, 34270 Saint-Mathieu-de-Tréviers - prenom.nom@hydris-hydrologie.fr \\ Irstea, UR HHLY, Hydrologie-Hydraulique, 5 rue de la Doua CS70077,69626Villeurbanne Cedex - prenom.nom@irstea.fr
}

\begin{abstract}
RÉSUMÉ. - Cette communication présente une synthèse des différents outils qui ont été utilisés pour consolider la courbe de tarage pour des crues d'occurrence rare du Réal Collobrier au Pont de Fer (Pierrefeu-du-Var). Du 10 au 14 novembre 2011 le bassin du Réal Collobrier a été touché par une crue qui a duré plus de 5 jours. La cote maximale enregistrée lors de cet événement $(4,3 \mathrm{~m})$ dépasse de $2 \mathrm{~m}$ le domaine de validité de la courbe de tarage en vigueur. Deux approches sont présentées. Une première approche a consisté à réaliser une campagne de mesure (relevés de terrain) pour caler un modèle hydraulique et définir les a priori sur les écoulements. Grâce à la méthode bayésienne BaRatin, une courbe de tarage avec un intervalle d'incertitude a été construite à partir des jaugeages avec leurs incertitudes et des a priori déterminés avec le modèle hydraulique. La seconde approche est basée sur la modélisation hydrologique du bassin versant. Le modèle conceptuel semi distribué, GR3H, a permis de modéliser l'hydrogramme de crue au pas de temps horaire à partir de pluie de bassin. Ces dernières ont été construites à partir des mesures enregistrées par les pluviographes lors de l'évènement. Les résultats montrent que les deux approches sont cohérentes concernant le volume de crue et la forme de l'hydrogramme, mais pas sur la valeur du débit moyen horaire maximum. Enfin une estimation de la période de retour de cet événement a pu être déterminée par la méthode Shyreg débit.
\end{abstract}

Mots-clés : prévision crue, modèle hydraulique, BaRatin, Shyreg

\section{Consolidation method for rating curves in case of rare floods in the Réal Collobrier experimental watershed}

\begin{abstract}
This paper presents an overview of the different tools used to extend the rating curve to rare floods on the Réal-Collobrier located in Pierrefeu-du-Var, Southern France. From the $10^{\text {th }}$ to the $14^{\text {th }}$ of November 2011, this catchment was affected by a flood that lasted more than 5 days. The highest water level recorded during this event $(4.3 \mathrm{~m})$ exceeded by 2 meters the area of validity of the current rating curve. Two approaches are presented. The first methodology relies on a field survey to calibrate a hydraulic model and determine the priors on water flows. The Bayesian method BaRatin led to estimate a rating curve and its uncertainty interval using the river gauging measurements and the modeled water flows derived from the hydraulic model. The second approach relies on the hydrologic modeling of the watershed. The semi-distributed conceptual model GR3H was forced by catchment rainfall, which were estimated from rain gauges measurements, to produce the hourly flood hydrograph. The results show that both approaches are consistent regarding flood volume and hydrograph shape but aren't on maximum hourly mean discharge. To conclude, we provide an estimation of the return period of this event based on the Shyreg-discharge methodology.
\end{abstract}

Key-words: Flood forecast, hydraulic modeling, BaRatin, Shyreg

\section{INTRODUCTION}

Le bassin versant expérimental du Réal Collobrier est situé sur la façade méditerranéenne dans le département du Var, à l'extrémité Ouest du massif montagneux des Maures, à $20 \mathrm{~km}$ au Nord-Est de Toulon (Lavabre et al.). Avec 17 postes pluviographiques et 11 stations de contrôle des débits répartis sur $70 \mathrm{~km}^{2}$, il constitue un site repère de l'hydro-climatologie régionale depuis 1966 (Marc et al., 1995).

Du 10 au 14 novembre 2011, le département du Var a subi d'importantes précipitations (Collombat, 2012). Le bassin du Réal Collobrier a été particulièrement impacté par cet épisode. Les particularités de cet évènement sont sa durée importante (5 jours) et la constance de son intensité horaire $(5 \mathrm{~mm} / \mathrm{h})$

Ainsi, le bassin versant s'est saturé progressivement et a répondu fortement aux dernières pluies qui se sont focalisées sur la partie du relief amont du bassin. Sur la plupart des stations hydrométriques, les enregistrements des cotes ont dépassé le domaine de validité des courbes de tarage (Valescure, Maurets, Maliére, Collobriéres, Pont de Fer). Certaines stations ont été contournées ou des enregistreurs noyés. Cet événement présente le record des hauteurs d'eau enregistrées en 45 ans de mesure sur les stations limnimétriques ; il dépasse la crue de référence de septembre 1968. 


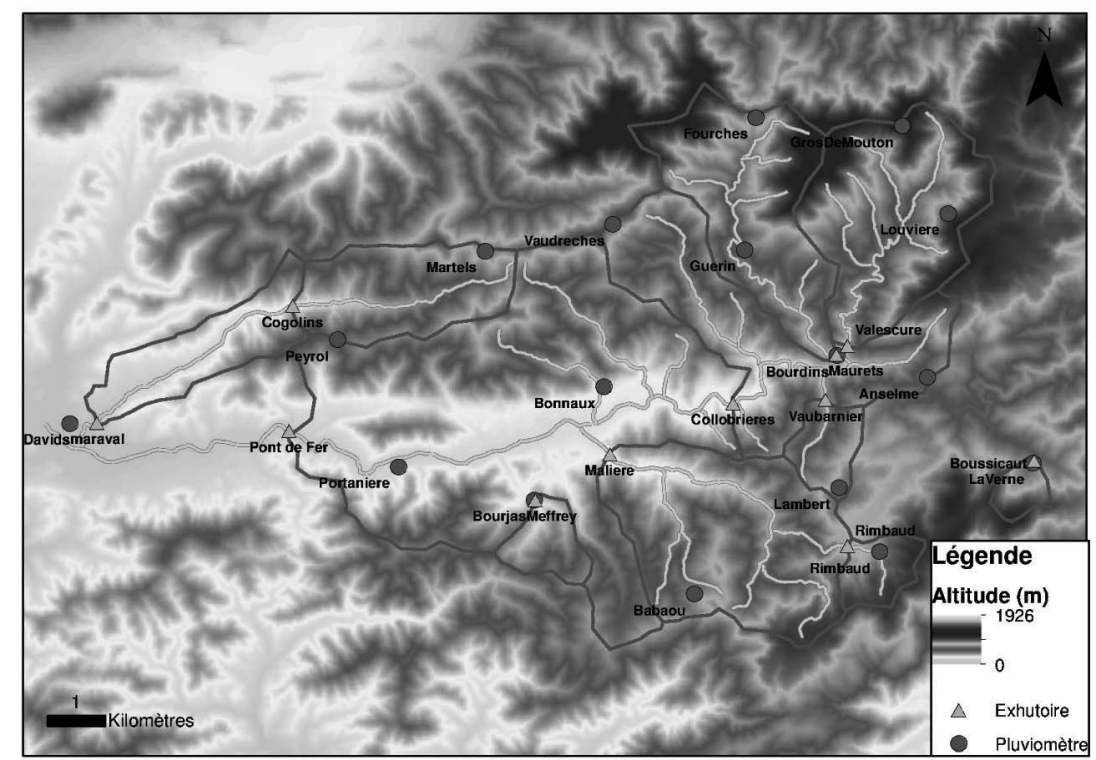

Figure 1 : Le bassin versant expérimental du Réal Collobrier avec les sous bassins versants, les triangles rouges correspondent aux 11 stations limnimétriques et les points verts aux 17 pluviographes.

La station la plus touchée est celle située à l'exutoire du bassin versant : la station Pont de Fer, à Pierrefeu-du-Var. La Figure 2 à gauche représente la courbe de tarage $n^{\circ} 13$ anciennement utilisée. Elle est construite à partir d'une régression sur les débits jaugés (les points de couleurs roses). La Figure 2 à droite représente le limnigramme de crue enregistré par la station. Avec une hauteur d'eau enregistrée par le limnigraphe de 4,3 m, la limite de validité de la courbe de tarage a été dépassée de plus de $2 \mathrm{~m}$.

Afin de consolider la courbe de tarage pour des crues d'occurrence rare, nous présentons dans cet article différentes approches qui se classent en deux catégories :

- approche se basant uniquement sur l'information sur les débits : relevés des laisses de crue, relevés topographiques, calage d'un modèle hydraulique pour définir les a priori hydrauliques et mettre en ouvre de la méthode BaRatin.

- approche se servant d'autres variables explicatives : mise en œuvre d'un modèle pluie-débit semi distribué sur le bassin versant et estimation de l'occurrence de l'événement par l'approche Shyreg débit.

\section{APPROCHE PAR LES DÉBITS}

Dans cette partie est analysée la mise en place d'un modèle hydraulique grâce au retour d'expérience sur le terrain (laisses de crue, relevés topographiques), afin de déterminer des a priori sur les écoulements. C'est à partir de ces derniers et des jaugeages avec leurs incertitudes que BaRatin construira la nouvelle courbe de tarage avec les intervalles d'incertitudes à $95 \%$.

\section{II.1. Modélisation hydraulique}

Le site de Pont de Fer présente une section assez simple à modéliser (Figure 3). Les lits majeurs droit et gauche sont larges d'une dizaine de mètres chacun. Le Pont de Fer a un
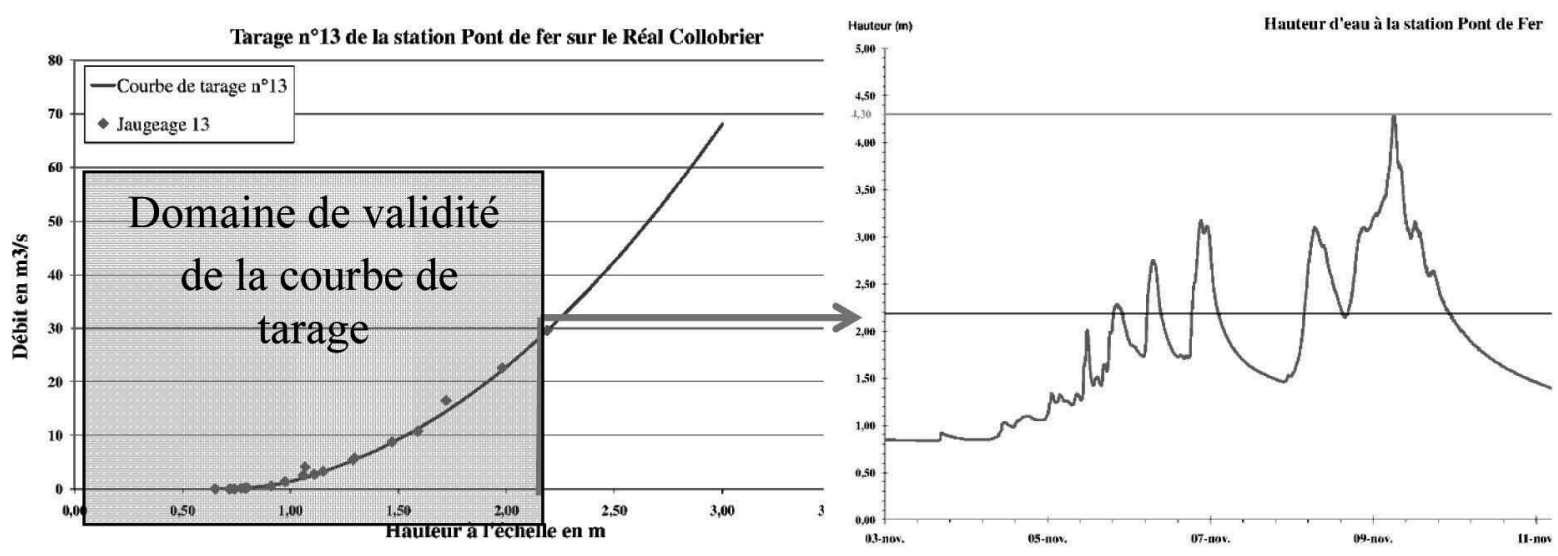

Figure 2 : À gauche la courbe en bleu représente la courbe de tarage $n^{\circ} 13$ de la station Pont de Fer avant l'événement de novembre 2011. Les points roses représentent les débits jaugés. À droite la courbe rouge représente les hauteurs d'eau enregistrées à la station Pont de Fer pendant l'évènement de novembre 2011. La droite bleue montre la limite de validité de la courbe de tarage $n^{\circ} 13$ et la droite verte la hauteur maximale de $4.3 \mathrm{~m}$ enregistrée lors de l'évènement. 


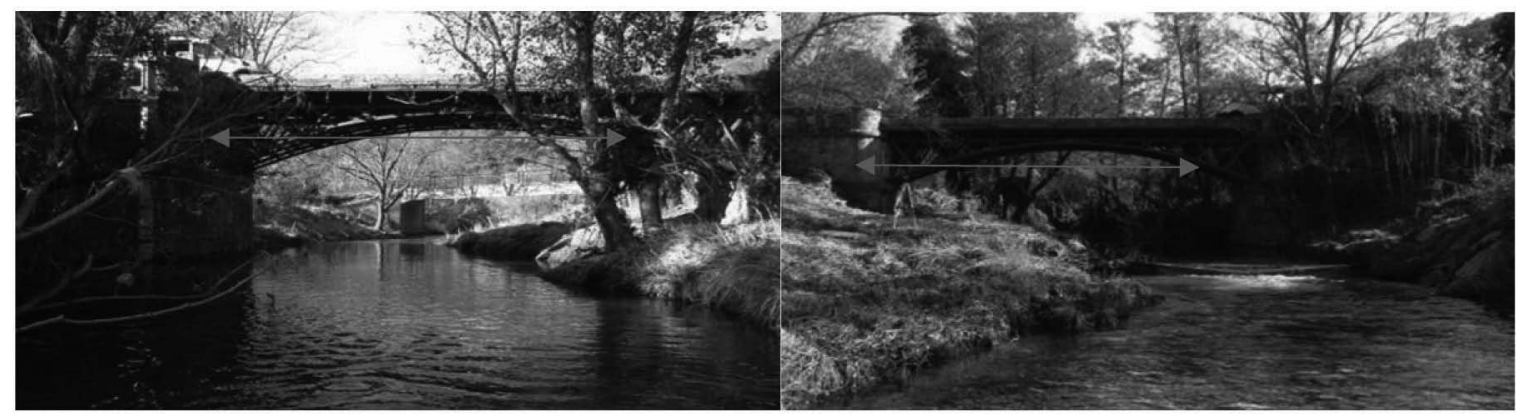

Figure 3 : Photo à gauche prise en amont du Pont de Fer (à gauche l'échelle limnimétrique), à droite photo du Pont de Fer depuis l'aval avec le seuil. Les flèches rouges montrent la cote maximum atteinte par l'écoulement lors de la crue.

tablier long de $14 \mathrm{~m}$ offrant une section importante à l'écoulement. Le seuil permettant de contrôler les débits est situé à l'aval du Pont de Fer. En aval du seuil la pente hydraulique devient plus importante. Le second pont situé en aval du seuil n'est pas modélisé car il a très peu d'impact sur l'écoulement (section d'écoulement plus importante que celle du Pont de Fer) .

Les laisses de crue, en amont du pont, ont montré que la ligne d'eau n'est descendue que de quelques centimètres laissant penser que le régime critique ne s'est pas établi et que le pont ne s'est pas mis en charge (l'eau a atteint les arches métalliques mais pas le tablier du pont). La ligne d'eau maximum lors de l'événement du 11 novembre 2011 est située à 4,3 $\mathrm{m}$ au dessus du seuil (sur la Figure 3, à gauche, les laisses de crue sont aperçues dans les arbres et la flèche rouge montre la cote). La hauteur d'eau maximale enregistrée par le limnigraphe correspond bien à celle indiquée par les laisses de crue.

Le lit majeur a contenu entièrement l'écoulement en crue permettant d'avoir un bief plutôt simple à modéliser. Les ponts ont eu peu d'impact sur les écoulements, à part le fait d'obstruer localement le lit majeur. La campagne de terrain a permis de constituer 10 sections transversales, sur un linéaire total d'une cinquantaine de mètres, avec au milieu le Pont de Fer et le seuil. Pour chaque profil en travers trois zones ont été définies avec des coefficients de Strickler différents afin d'optimiser les couples hauteur-débit entre le modèle hydraulique et les observations (Viollet et al., 2003):

- le lit mineur sans végétation : coefficient de Strickler fixé à $40 \mathrm{~m}^{1 / 3} / \mathrm{s}$
- le lit moyen composé d'herbes : coefficient de Strickler fixé à $30 \mathrm{~m}^{1 / 3} / \mathrm{s}$

- le lit majeur composé d'arbres et de buissons : coefficient de Strickler fixé à $10 \mathrm{~m}^{1 / 3} / \mathrm{s}$

Le modèle hydraulique utilisé est HEC-RAS. C'est un modèle unidimensionnel en régime permanent. Les conditions aux limites sont en aval la pente moyenne lorsque l'écoulement est fluvial et si l'écoulement est torrentiel la condition en amont est la hauteur critique. La Figure 4 représente la courbe de tarage issue de la modélisation hydraulique pour une gamme de couple de débit $\left(0,1\right.$ à $\left.200 \mathrm{~m}^{3} / \mathrm{s}\right)$ et de hauteur $(0$ à $4,5 \mathrm{~m})$. La hauteur limite de la courbe de tarage correspond à $50 \mathrm{~cm}$ de tirant drair sous le tablier du pont. Sur la Figure 4 on représente la courbe de targe $n^{\circ} 13$ qui est issue d'une régression de type puissance à partir de débits jaugés (points de couleur rouge).

Les résultats des modélisations des écoulements avec le modèle hydraulique 1D HEC-RAS montrent pour les faibles débits $\left(<30 \mathrm{~m}^{3} / \mathrm{s}\right)$ :

- une bonne adéquation du couple hauteur-débit entre le modèle HEC-RAS et les jaugeages

— un régime critique établi au droit du seuil

— pour les débits supérieurs à $10 \mathrm{~m}^{3} / \mathrm{s}$, le seuil ne fait plus contrôle à l'écoulement

- sur la courbe de tarage issue de la modélisation hydraulique, une « cassure » est à noter, pour une hauteur de 2,5 m, correspondant à la cote de mise en eau du lit moyen. Alors que l'ancienne courbe de tarage, ajustée sur les débits jaugés, ne montre pas cette cassure. Ce point est important car

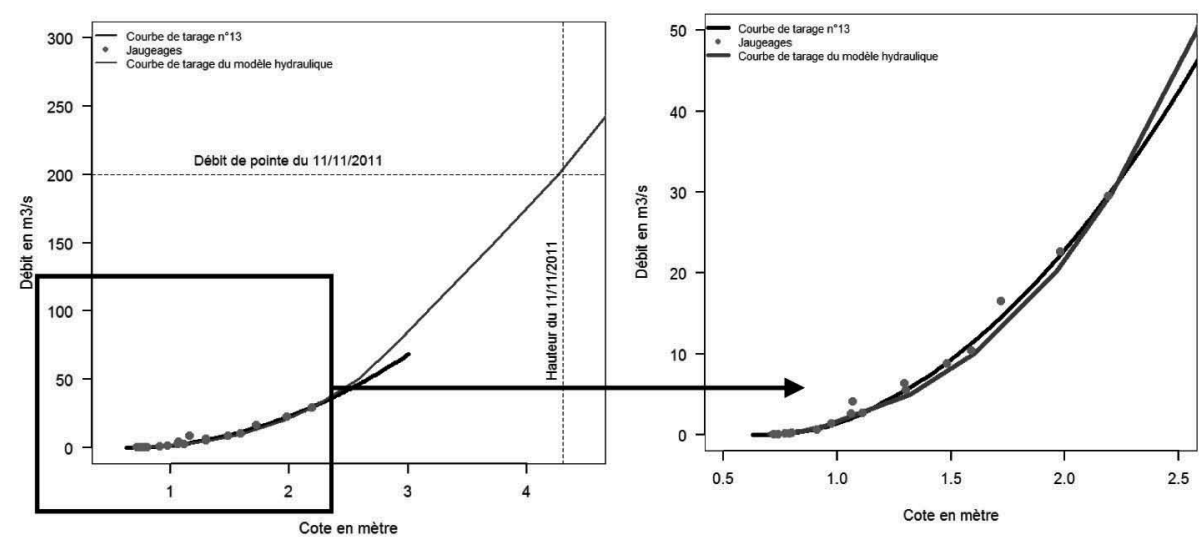

Figure 4 : Courbe de tarage issue de la modélisation hydraulique au droit du seuil (courbe bleue), courbe de tarage $n^{\circ} 13$ issue d'une régression sur les débits jaugés (courbe noire) et les points rouges représentent les débits jaugés effectués lors de la dernière campagne de crue entre 1992 et 1996. 
en réalité, il y a bien une augmentation « brusque » de section lors de la mise en eau du lit moyen .

Pour des hauteurs supérieures à $1,5 \mathrm{~m}$, le régime critique n'est plus établi au droit du seuil (le seuil es noyé). L'écoulement reste fluvial en amont et en aval du seuil.

La crue du mois de novembre 2011 est estimée à un débit de $200 \mathrm{~m}^{3} / \mathrm{s}$. La Figure 5 montre la ligne d'eau (en bleu) et la hauteur critique (en rouge) modélisées par HEC-RAS pour un débit de $200 \mathrm{~m}^{3} / \mathrm{s}$.

Pour un débit de $200 \mathrm{~m}^{3} / \mathrm{s}$ plusieurs remarques concordent avec nos observations :

- le régime critique n'est pas atteint à l'entrée du pont

- le pont ne s'est pas mis en charge, il reste $1 \mathrm{~m}$ de tirant d'air

- les vitesses moyennes d'écoulement sont de l'ordre de $4 \mathrm{~m} / \mathrm{s}$

— un débit pseudo spécifique de $6,7 \mathrm{~m}^{3} / \mathrm{s} / \mathrm{km}^{1.6}$ (Payrastre, 2005; Yoo et al., 2007) : cette valeur est comparable, au débit enregistré sur le bassin de Malière, $7.3 \mathrm{~m}^{3} / \mathrm{s} / \mathrm{km}^{1.6}$, pour le même évènement (voir Figure 1 pour l'emplacement de ce bassin)

\section{II.2. Analyse bayésienne par la méthode BaRatin}

Les a priori sur les écoulements ont été définis grâce au modèle hydraulique HEC-RAS. Cette modélisation a permis de préciser les transitions entre les contrôles hydrauliques successifs. Une base physique est fournie pour l'extrapolation de la courbe de tarage dans des gammes où les crues ne sont pas jaugées.

La méthode BaRatin va permettre d'estimer la courbe de tarage et les incertitudes associées à l'aide d'un formalisme bayésien (Branger et al., 2012). BaRatin se sert de l'ensemble des jaugeages, avec des incertitudes sur les débits jaugés (communément fixé à $7 \%$ ) à la fois en basses eaux et en hautes eaux, et des connaissances des contrôles hydrauliques, afin de construire une courbe de tarage avec un intervalle d'incertitude.

Pour chacune des gammes de hauteur, h, la méthode exprime le débit avec la formulation : $Q=a \cdot(h-b)^{c} \mathrm{Le}$ coefficient $\mathrm{c}$ est fonction du type de contrôle. Dans le cas d'un contrôle hydraulique de type Manning Strickler (écoulement fluvial), $\mathrm{c}=1,65$ et dans le cas d'un contrôle hydraulique de type seuil ou déversoir, $\mathrm{c}=2.5$.

Les coefficients, a et $b$, ont été optimisés de façon à reproduire les débits jaugés et modélisés selon les gammes de hauteur. Pour les faibles hauteurs, a et b ont été optimisés pour reproduire au mieux le couple débit jaugé-hauteur et pour les hauteurs où nous avons pas de débits jaugés, $\mathrm{a}$ et $\mathrm{b}$ sont calés pour reproduire au mieux le couple hauteur-débit issu du modèle hydraulique. Le Tableau 1 synthétise les résultats à la fois sur les a priori (le coefficient c) et les résultats du calage des coefficients a et $b$.

La Figure 4 montre la courbe de tarage issue des simulations de BaRatin au droit du seuil pour des hauteurs comprises entre 0 et $4,5 \mathrm{~m}$.

La courbe de tarage modélisée par BaRatin, pour les basses eaux, reproduit correctement les jaugeages. Pour les hauteurs comprises entre 3 et $4 \mathrm{~m}$, le modèle hydraulique fournit, des débits plus forts que BaRatin.

Pour la crue de novembre 2011, avec une hauteur de 4,3 $\mathrm{m}$, cette courbe de tarage estime un débit de pointe de $176 \mathrm{~m}^{3} / \mathrm{s}$ et en tenant compte de l'intervalle d'incertitude, le débit de pointe, est compris entre $206 \mathrm{~m}^{3} / \mathrm{s}$ et $158 \mathrm{~m}^{3} / \mathrm{s}$.

Le débit de pointe estimé pour la plus forte crue par BaRatin est moins fort que celui fourni par le modèle hydraulique. Néanmoins, la valeur de $200 \mathrm{~m}^{3} / \mathrm{s}$ estimé par ce dernier est contenue dans l'intervalle d'incertitude, ce qui conforte la courbe de tarage en hautes eaux. L'hydrogramme de crue qui sera utilisé pour la comparaison avec la

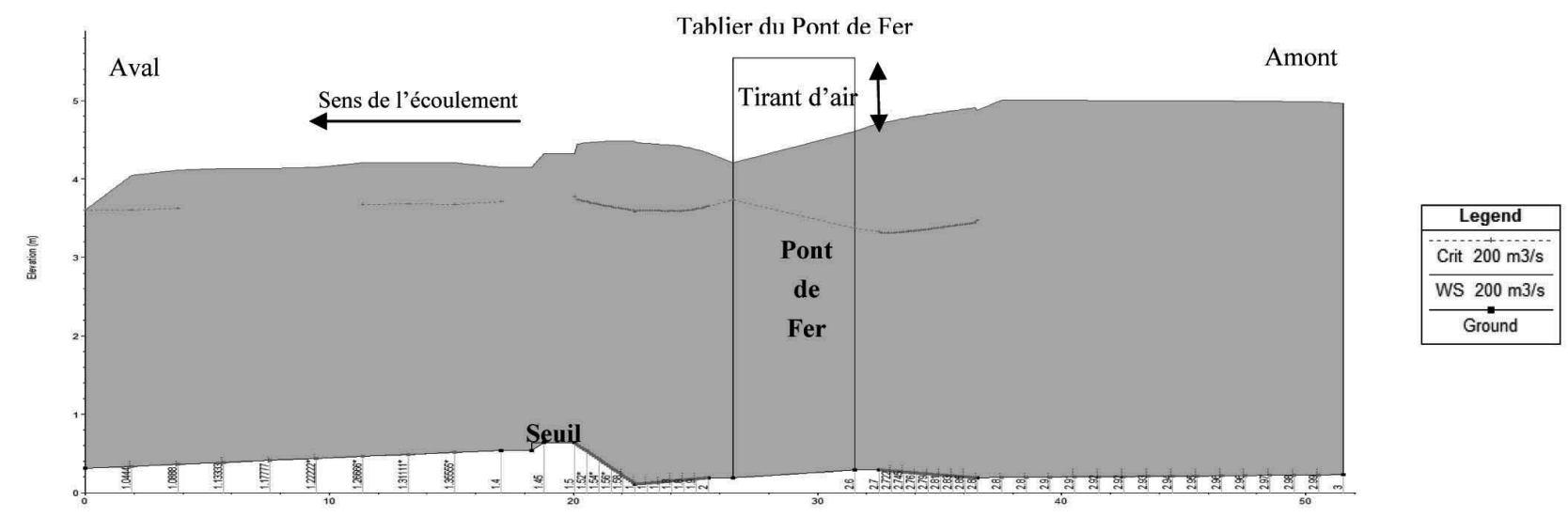

Figure 5 : Coupe longitudinale du site "Pont de Fer » avec 10 sections transversales levées sur sites et les lignes d'eau modélisées avec un débit de $200 \mathrm{~m}^{3} / \mathrm{s}$.

Tableau 1 : Caractéristiques de l'écoulement et des coefficients $a$, $b$ et c utilisés pour caler BaRatin

\begin{tabular}{|c|c|c|c|c|c|}
\hline Hauteurs & Débits en $\mathbf{m}^{3} / \mathbf{s}$ & Régime & Coefficients & Sections & Lois \\
\hline $\mathrm{H}<1 \mathrm{~m}$ & $\mathrm{Q}<1.7 \mathrm{~m}^{3} / \mathrm{s}$ & Critique & $\mathrm{a}=19.45 \quad \mathrm{~b}=0.63 \quad \mathrm{c}=2.5$ & $\mathrm{~S}<1.4 \mathrm{~m}^{2}$ & \multirow{3}{*}{$Q=a \cdot(h-b)^{c}$} \\
\hline $1<\mathrm{H}<2.45 \mathrm{~m}$ & $1.7<\mathrm{Q}<10 \mathrm{~m}^{3} / \mathrm{s}$ & Fluvial & $a=16.1 \quad b=0.75 \quad c=1.65$ & $1.4<\mathrm{S}<6 \mathrm{~m}^{2}$ & \\
\hline $\mathrm{H}>2.45 \mathrm{~m}$ & $10<\mathrm{Q}<250 \mathrm{~m}^{3} / \mathrm{s}$ & Fluvial & $\mathrm{a}=15.2 \quad \mathrm{~b}=2.45 \quad \mathrm{c}=1.65$ & $\mathrm{~S}>6 \mathrm{~m}^{2}$ & \\
\hline
\end{tabular}




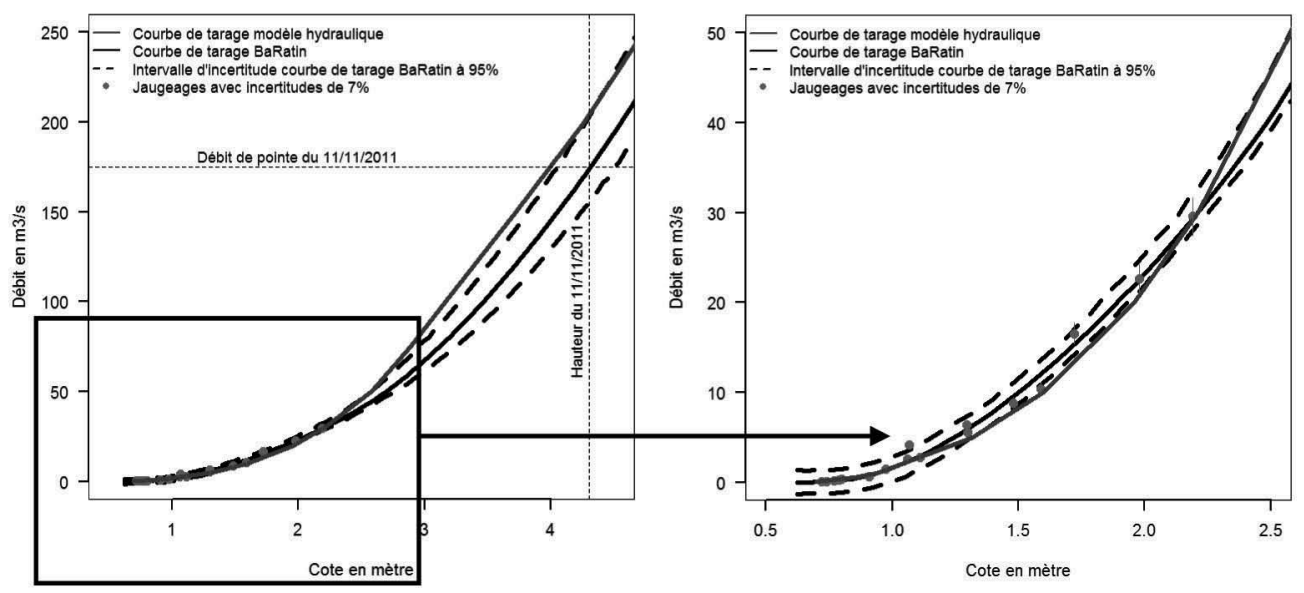

Figure 6 : Courbe de tarage et enveloppe des incertitudes à $95 \%$ issues de la simulation de BaRatin pour les faibles et les hautes eaux au droit du seuil et les points représentent les débits jaugés avec leurs incertitudes.

modélisation hydrologique est issu de cette courbe de tarage. Par la suite, les débits sont moyennés sur le pas de temps horaire et le débit moyen horaire maximal de la crue de novembre 2011 est estimé à $160 \mathrm{~m}^{3} / \mathrm{s}$.

\section{APPROCHE PAR LES MODELES HYDROLOGIQUES ET ESTIMATION DE L'OCCURRENCE DE L'EVENEMENT}

\section{III.1. Modélisation hydrologique semi distribuée}

Cette partie va nous permettre de comparer les débits issus de la modélisation hydrologique et ceux de BaRatin. A cet effet, nous avons mis en place un modèle pluie-débit semidistribué au pas de temps horaire. Les sorties de la modélisation, c'est-à-dire la forme de l'hydrogramme de crue, le débit moyen horaire (proche d'un débit de pointe) et le volume sont comparés à l'hydrogramme de crue issu de la courbe de tarage de BaRatin. La mise en œuvre d'une modélisation semi distribuée a été préférée à une modélisation globale, après examen de la répartition spatiale des champs de pluies. Avant que se produise le plus grand pic de crue, on constate que les intensités de pluie les plus importantes se sont concentrées dans la partie nord-Est du bassin versant. Une modélisation globale aurait lissé de façon trop importante la pluie de bassin, crest pourquoi le bassin versant de la station Pont de Fer $\left(69,7 \mathrm{~km}^{2}\right)$ a été divisé en trois sous-bassins : Valescure $\left(9,3 \mathrm{~km}^{2}\right)$, Maurets $\left(8,4 \mathrm{~km}^{2}\right)$ et Pont de Fer Restant $\left(52 \mathrm{~km}^{2}\right)$.

\section{III.1.1. Pluie}

Une pluie de bassin est construite sur chacun des 3 sous bassins (Valescure, Maurets et Pont de Fer Restant, dont les caractéristiques sont présentées dans le Tableau 2) à partir des 17 pluviographes disponibles sur le bassin expérimental du Réal Collobrier (Figure 7 à gauche). Les pluies de bassin ont été calculées par combinaison linéaire de valeurs aux postes sélectionnés, avec des pondérations issues de la méthode des polygones de Thiessen. La somme de ces pondérations est égale à 1 sur chacun des sous bassins. Lors de l'événement du mois de novembre 2011 on estime des cumuls de pluie de bassin en $72 \mathrm{~h}$ de $440 \mathrm{~mm}$ (Maurets), $364 \mathrm{~mm}$ (Valescure) et $310 \mathrm{~mm}$ (Pont de Fer Restant).
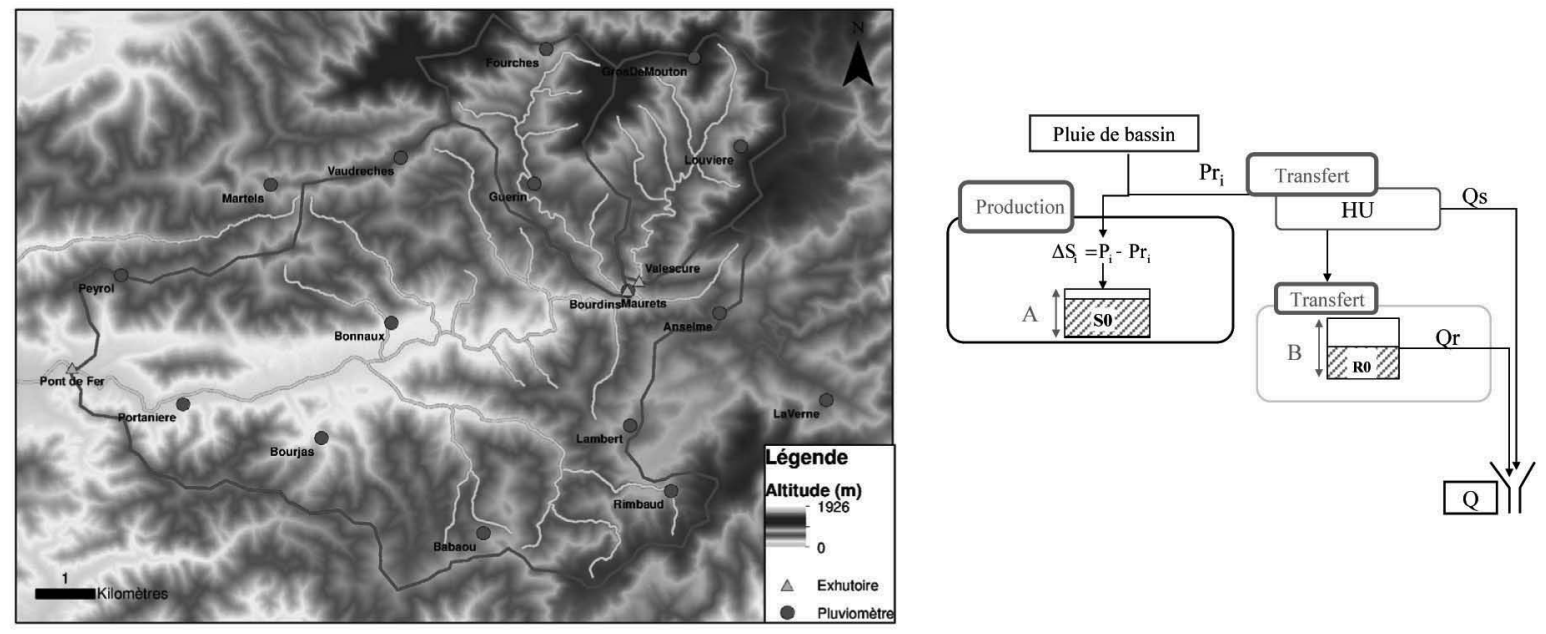

Figure 7 : à gauche, emplacement des 3 sous bassins versants (Valescure, Maurets et Pont de Fer Restant), avec leurs pluviographes et leurs stations hydrométriques et à droite, structure du modèle GR3H. 
Tableau 2: Valeurs des paramètres servant à la modélisation hydrologique

\begin{tabular}{|l|c|c|c|c|c|c|c|}
\hline \multicolumn{1}{|c|}{ Bassins } & Surface $\left.\mathbf{( k m}^{2}\right)$ & Pluviograhes & A (mm) & S0 (mm) & B (mm) & R0 (mm) & HU (h) \\
\hline $\begin{array}{l}\text { Pont de Fer } \\
\text { restant }\end{array}$ & 52 & 11 & 369 & 37 & 224 & 48 & 3 \\
\hline Valescure & 9.34 & 2 & 369 & 37 & 112 & 39 & 4 \\
\hline Maurets & 8.4 & 3 & 369 & 37 & 112 & 38 & 4 \\
\hline
\end{tabular}

\section{III.1.2. Modèle hydrologique}

Le modèle hydrologique utilisé sur chacun des sous bassins versants (GR3H Figure 7 à droite) est un modèle conceptuel à 2 réservoirs fonctionnant au pas de temps horaire (Yang, 1993). Le premier réservoir (A) gère la production et assure une production non-linéaire entre la pluie stockée dans ce même réservoir et la pluie routée vers le réservoir de transfert (B). La taille de ce premier réservoir est imposée à $369 \mathrm{~mm}$, valeur unique qui est utilisée dans la méthode Shyreg sur tous les bassins du Réal Collobrier (Aubert, 2012).

La taille du second réservoir, la durée des hydrogrammes unitaires (HU) ainsi que les états initiaux des réservoirs de production ( $\mathrm{S} 0$ ) et de transfert (R0), ont été optimisés sur chacun des trois sous-bassins pour que la somme des 3 hydrogrammes puisse reproduire au mieux la première partie de l'hydrogramme de crue du 10 novembre 2011 où les cotes enregistrées restent inférieures à $2,5 \mathrm{~m}$.

La faible valeur du coefficient d'humidité $\left(\mathrm{S}_{0} / \mathrm{A}\right)$ est cohérente avec la longue période sèche antérieure (mai-octobre 2011). Les tailles des réservoirs (B) sont dans les ordres de grandeur de celles obtenues lors d'études précédentes (Arnaud, 2005).

\section{III.1.3. Modélisation de la crue de novembre 2011 au Pont de Fer}

La mise en œuvre d'une modélisation semi-distribuée a été nécessaire car la dernière pointe de l'hydrogramme de la crue au Pont de Fer résulte des crues issues des sous-bassins situés en amont et sur le relief du Réal Collobrier.. Un décalage temporel entre la pluie et le débit variant de $2 \mathrm{~h}$ à $4 \mathrm{~h}$, suivant l'emplacement des sous-bassins par rapport à l'exutoire, a été pris en compte.
La Figure 8 à gauche montre le taux de saturation du réservoir de production sur le bassin versant de Valescure. À droite la modélisation hydrologique des 3 sous bassins (Valescure, Maurets et Pont de Fer Restant). Par addition des 3 hydrogrammes de crue, on obtient l'hydrogramme de crue à l'exutoire (Figure 8 à droite). Les modèles hydrologiques ont été calibrés pour que l'hydrogramme de crue à l'exutoire reproduise au mieux le débit observé issu de la courbe de tarage BaRatin, jusqu'à $\mathrm{t}=105 \mathrm{~h}$. Nous laissons, pour la seconde partie de l'événement, GR3H modéliser « librement », à paramétrage constant.

Cette crue est intéressante, du point de vue hydrologique, car elle a commencé sur des sols relativement secs qui se sont saturés ensuite en raison des pluies tombées en continu durant 5 jours. Les dernières pluies se sont concentrées en amont du bassin et sur le relief alors qu'en plaine, les précipitations étaient moindres.

Les niveaux de remplissage du réservoir de production, pour les durées comprises entre $t=118 \mathrm{~h}$ et $\mathrm{t}=180 \mathrm{~h}$, sont très élevés, montrant une saturation complète du bassin versant (pour le bassin de Valescure, $\mathrm{S} 0 / \mathrm{A}=0,94$ à $\mathrm{t}=140 \mathrm{~h}$, Figure 8 à gauche). Les dernières pluies avec des intensités horaires comprises entre 15 et $25 \mathrm{~mm} / \mathrm{h}$, qui peuvent paraître faibles, arrivant sur des sols saturés ont provoqué le dernier pic de crue.

L'hydrogramme de crue du bassin versant de Valescure modélisé, sur la Figure 8 à droite, montre un débit moyen horaire de $46 \mathrm{~m}^{3} / \mathrm{s}(\mathrm{t}=150 \mathrm{~h})$. Soit un débit pseudo spécifique de $7.7 \mathrm{~m}^{3} / \mathrm{s} / \mathrm{km}^{1.6}$, valeur qui est conséquente et cohérente avec celle du bassin versant de Malière.

L'examen de la Figure 8 à droite montre le résultat de la modélisation hydrologique (courbe rouge). L'hydrogramme de crue issu de l'addition des 3 hydrogrammes (Valescure, Maurets et Pont de Fer Restant) pour la période de calage
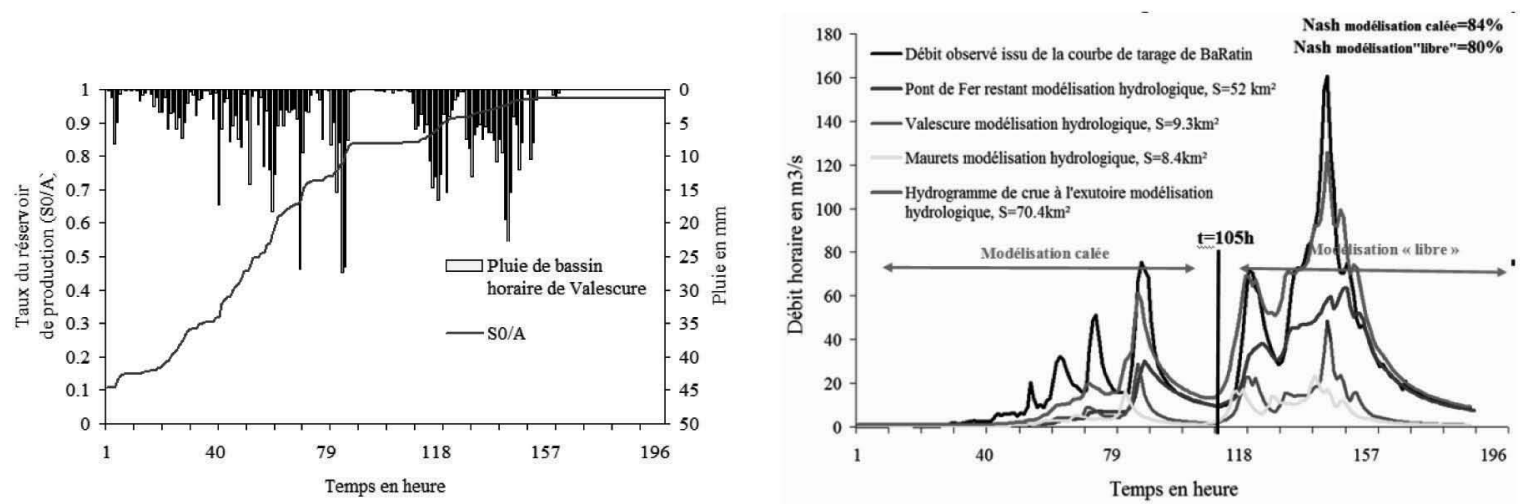

Figure 8 : à gauche modélisation du taux d'eau dans le réservoir de production du bassin versant de Valescure au cours de l'évènement pluvieux et à droite l'hydrogramme de crue des sous-bassins du Réal Collobrier et estimation de l'hydrogramme de crue au Pont de Fer à partir de la modélisation hydrologique. 
permet de modéliser seulement le dernier pic de crue $(\mathrm{t}=88 \mathrm{~h})$. Ce constat vient :

— du critère de calage qui est le critère de Nash : ce dernier a un poids plus important sur les fortes valeurs que sur les plus faibles ce qui explique sa valeur pour la période de calage de $84 \%$

- la durée de l'évènement : la période de calage s'étalant sur plus de 5 jours, le modèle cale les états initiaux des réservoirs de transfert et de production suffisamment bas pour refaire le dernier de pic de crue. En effet compte tenu de la structure du modèle, une bonne reproduction des deux premiers pics de crues (entre $\mathrm{t}=50$ et $60 \mathrm{~h}$ ) aurait eu pour conséquence une saturation des réservoirs trop anticipée et une surestimation des débits lors de l'assimilation des précipitations suivantes.

Pour la période où le modèle est " libre ", la forme de l'hydrogramme de crue est respectée. Le critère de Nash estimé entre l'hydrogramme observé et l'hydrogramme modélisé est bon. Il est de $80 \%$. Les débits moyens journaliers modélisés et observés, calculés entre les pas de temps $134 \mathrm{~h}$ et $158 \mathrm{~h}$, sont très proches : $87 \mathrm{~m}^{3} / \mathrm{s}$ modélisés et $85 \mathrm{~m}^{3} / \mathrm{s}$ observé. Ce volume important est la plus forte valeur enregistrée depuis l'ouverture de la station.

Toutefois, la modélisation hydrologique ne permet pas de reproduire le plus fort débit moyen horaire de l'hydrogramme $\left(160 \mathrm{~m}^{3} / \mathrm{s}\right.$ observé contre $123 \mathrm{~m}^{3} / \mathrm{s}$ modélisé). Soit un écart de $23 \%$. La structure du modèle peut expliquer cet écart. L'obtention du pic de crue est dépendante du décalage temporel entre les sous-bassins versants (4 h Valescure et Mauret et $3 \mathrm{~h}$ pour Pont de Fer Restant). Ce dernier est fixe pour tout l'évènement alors que l'on pourrait imaginer une cinétique différente selon l'intensité de la crue.

\section{III.2. Estimation de l'occurrence de l'événement par la méthode Shyreg débit}

Nous avons déterminé à partir de BaRatin une nouvelle courbe de tarage. Lors de l'événement du 11 novembre 2011, nous estimons avec la nouvelle courbe un débit de pointe de $174 \mathrm{~m}^{3} / \mathrm{s}$ et un débit moyen journalier de $85 \mathrm{~m}^{3} / \mathrm{s}$. Dans ce paragraphe, est estimée l'occurrence d'un tel événement en terme de période de retour à l'aide de la méthode Shyreg-débit.

La méthode Shyreg couple un générateur stochastique de pluie horaire et un modèle hydrologique régionalisés aux $\mathrm{km}^{2}$ sur l'ensemble du territoire français (Arnaud et al., 2002; Aubert, 2012; Organde et al., 2013). La méthode Shyreg débit a été mise en oeuvre sur le bassin du Réal Collobrier. La méthode a été recalée sur les quantiles 2, 5 et 10 ans issus d'une loi statistique de la théorie des valeurs extrêmes (loi GEV) ajustée aux débits maxima annuels de pointe et journaliers observés entre 1966 et 2011. Les débits sont estimés à l'aide de la courbe de tarage issue de BaRatin. La Figure 9 représente les distributions de fréquence : des quantiles de débit Shyreg, des quantiles GEV et les débits de pointe et moyens journaliers observés sur la période 1966-2011.

Shyreg débit reproduit bien les distributions de fréquence des débits de pointe et des débits moyens journaliers observés. L'analyse de l'épisode du mois de novembre 2011 montre que les débits de pointe et moyens journaliers de crue sont les plus forts de la chronique. On note que la méthode Shyreg débit attribue une fréquence d'apparition plus importante sur le débit moyen journalier (75 ans) que sur les débits de pointe (60 ans).

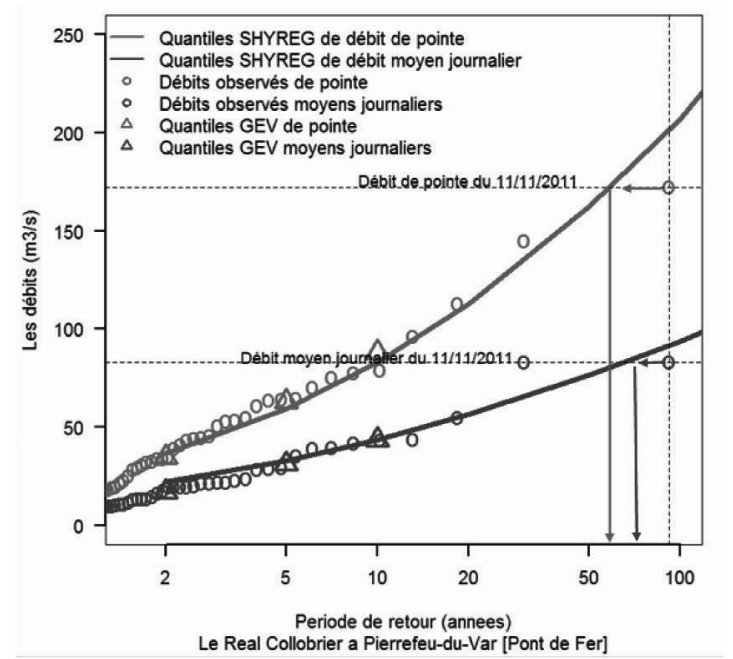

Figure 9 : Distribution de fréquence des débits sur le bassin du Réal Collobrier au Pont de Fer avec la méthode Shyreg débit.

\section{CONCLUSIONS}

Nous avons proposé une méthodologie permettant de consolider une courbe de tarage pour les crues d'occurrence rare, ici appliquée à la station Pont de Fer sur le bassin du Réal Collobrier. Par rapport à la méthode classique de construction de courbe de tarage, c'est-à-dire une régression ajustée sur les débits jaugés, nous avons mis en œuvre plusieurs outils :

- un modèle hydraulique nous a permis de construire une première courbe de tarage et définir les a priori. Nous notons une très bonne adéquation entre les couples débit/ hauteur des observations et du modèle hydraulique .

- à partir des a priori définis par le modèle hydraulique, des jaugeages et de leurs incertitudes, nous avons mis en œuvre le logiciel BaRatin. Ce dernier nous a construit une nouvelle courbe de tarage (domaine de validité de 0 à $4,5 \mathrm{~m}$ ) avec un intervalle dsincertitude. La nouvelle courbe de tarage reproduit bien les débits jaugés et pour les hautes eaux, nous retrouvons le comportement asymptotique du modèle hydraulique. Avec la nouvelle courbe de tarage, pour une hauteur de $4,3 \mathrm{~m}$, le débit de pointe est estimé à $174 \mathrm{~m}^{3} / \mathrm{s}$ avec un intervalle d'incertitude sur les débits variant de 158 à $206 \mathrm{~m}^{3} / \mathrm{s}$.

- la mise en cuvre du modèle hydrologique GR3H nous a permis de comparer la forme de l'hydrogramme de crue résultant de la nouvelle courbe de tarage et de valider les volumes de la crue du mois de novembre 2011. Les résultats sur la forme et le volume de la crue sont bons. Néanmoins, en ce qui concerne les résultats sur les débits moyens horaires, (assimilable à des débits de pointe), le modèle hydrologique sous-estime ( $23 \%$ ) la valeur par rapport à celle issue de la nouvelle courbe de tarage.

Cette méthodologie va être étendue sur les autres stations du bassin versant expérimental du Réal Collobrier comme le Réal à Collobrières, Maliére et Maurets. D'autre part, l'utilisation lors de crues, des nouvelles méthodes d'exploration du champ de vitesses en surface des écoulements, comme les radars vélocimétriques, est à l'étude pour affiner un peu plus ces résultats. 


\section{REFERENCES}

ARNAUD P. (2005) - Simplification de GR3H pour la prédétermination des crues. Application sur des petits bassins versants : Rapport d'étude Cemagref, Aix-en-Provence. 26 pages

Aubert Y. (2012) - Estimation Des Valeurs Extremes De Debit Par La Methode Shyreg: Reflexions Sur L'equifinalite Dans La Modelisation De La Transformation Pluie-Debit. Thèse de Doctorat Université Pierre et Marie Curie (UPMC). 318 p

Branger F., J. Le Coz B. Renard \& L. Bonnifait (2012) - Méthode d'estimation des incertitudes sur les courbes de tarage, Fiche technique OTHU. 4 p

Collombat P.-Y. (2012) — Se donner les moyens de ses ambitions : les leçons des inondations du Var et du sud-est de la France. N. Rapport sénatorial, SÉNAT, SESSION EXTRAORDINAIRE DE. 2011-2012. Paris

LAVABRE J. \& V. MARC (1997) - Bassin versant de recherche du Réal Collobrier. Le rôle de la couverture forestière dans la lutte contre la désertification de la zone méditerranéenne. Incidence de la couverture forestière sur les eaux de pluie : aspects quantitatif et qualitatif. Rapport d'activité 1995-1996. 48 pages + figures

Marc V., Y. Travi \& J. Lavabre (1995) - Etude du fonctionnement hydrologique de bassins versants méditerranéens par le traçage naturel chimique et isotopique. AISH Publications. 229 : 219-229

Payrastre O. (2005) — Faisabilite Et Utilite Du Recueil De Donnees Historiques Pour L'etude Des Crues Extremes De Petits Cours D'eau. Etude Du Cas De Quatre Bassins Versants Affluents De L'aude. Thèse de Doctorat Ecole Nationale Des Ponts et Chaussées (ENPC). $390 \mathrm{p}$

Viollet P. L. \& J. P. Chabard (2003) - Mécanique des fluides appliquée Presses de l'Ecole Nationale des Ponts et Chaussées, Paris. 366 pages

YAng X. Mise (1993) — Au Point D'une Methode D'utilisation D'un Modele Pluie-Debit Conceptuel Pour La Prevision Des Crues. Ecole Nationale des Ponts et Chaussées (ENPC). 204 p

Yoo C., K. KIM H. S. KIM \& M. J. PARK (2007) - Estimation of areal reduction factors using a mixed gamma distribution. Journal of Hydrology. 335(3-4) : 271-284 